\title{
Educational status-related disparities in awareness, treatment and control of cardiovascular risk factors in India
}

\author{
Rajeev Gupta, ${ }^{1}$ Krishna Kumar Sharma, ${ }^{1}$ Bal Kishan Gupta, ${ }^{2}$ Arvind Gupta, ${ }^{3}$ \\ Revant R Gupta, ${ }^{4}$ Prakash C Deedwania ${ }^{5}$
}

\begin{abstract}
- Additional material is available. To view please visit the journal online (http://dx. doi.org/10.1136/heartasia2014-010551).

${ }^{1}$ Department of Medicine, Fortis Escorts Hospital, Jaipur, Rajasthan, India

${ }^{2}$ Department of Medicine, SP Medical College, Bikaner, India ${ }^{3}$ Jaipur Diabetes Research Centre, Jaipur, India

${ }^{4}$ Jaipur Heart Watch Foundation, Jaipur, India ${ }^{5}$ Department of Cardiology, University of California San Francisco, Fresno, California, USA
\end{abstract}

Correspondence to Dr Rajeev Gupta, Department of Medicine, Fortis Escorts Hospital, JLN Marg, Jaipur, Rajasthan 302017, India; rajeevgg@gmail.com

Received 15 June 2014 Revised 7 September 2014 Accepted 18 December 2014



To cite: Gupta $R$, Sharma KK, Gupta BK, et al. Heart Asia 2015;7:1-6. doi:10.1136/heartasia-2014010551

\section{ABSTRACT}

Objective To determine association of socioeconomic status, defined by educational status (ES), with awareness, treatment and control of cardiovascular risk factors.

Methods We performed an epidemiological study at 11 cities in India using cluster sampling. 6198 subjects (3426 men, 2772 women, response $62 \%$, age 48 \pm 10 years) were evaluated for sociodemographic, lifestyle, anthropometric and biochemical factors. ES was categorised according to years of schooling into low ( $\leq 10$ years), medium (11-15 years) and high ( $>15$ years). Risk factors were diagnosed according to current guidelines. Awareness, treatment and control status were determined for hypertension, diabetes and hypercholesterolaemia. For smoking/tobacco use, quit rate was determined. Descriptive statistics are reported.

Results Age-adjusted and sex-adjusted prevalence (\%) of various risk factors in low, medium and high ES subjects was hypertension 31.8, 29.5 and 34.1, diabetes 14.5, 15.3 and 14.3, hypercholesterolaemia 24.0, 23.9 and 27.3, and smoking/tobacco use 24.3, 14.4 and 19.0. Significantly increasing trends with low, medium and high ES were observed for hypertension awareness $(30.7,37.8,47.0)$, treatment $(24.3,29.2,35.5)$ and control $(7.8,11.6,15.5)$; diabetes awareness (47.2, $51.5,56.4)$, treatment $(38.3,41.3,46.0)$ and control $(18.3,15.3,22.8)$; hypercholesterolaemia awareness $(8.9,22.4,18.4)$, treatment $(4.1,6.2,7.9)$ and control $(2.8,3.2,6.9)$, as well as for smoking/tobacco quit rates $(1.6,2.8,5.5)\left(\chi^{2}\right.$ for trend, $\left.p<0.05\right)$.

Conclusions Low ES subjects in India have lower awareness, treatment and control of hypertension, diabetes and hypercholesterolaemia and smoking quit rates.

\section{INTRODUCTION}

Global Burden of Diseases Study (2010) has reported that mortality rates from major noncommunicable diseases such as cardiovascular diseases (CVD) are two to three times greater in lowincome than in high-income countries. ${ }^{1}{ }^{2}$ The Prospective Urban-Rural Epidemiology (PURE) study reported that age-adjusted annual cardiovascular mortality rates were $1.00 / 1000$ in highincome, 4.86/1000 in middle-income and 7.25/ 1000 in low-income countries despite the burden of cardiovascular risk factors being greater in highincome countries. ${ }^{3}$ This suggested that risk factor control and disease management is inferior in lower income countries. ${ }^{3}$ Multiple studies from high-income and middle-income countries have also reported that low-socioeconomic status subjects have greater all-cause as well as cardiovascular mortality compared with the middle-socioeconomic or high-socioeconomic status subjects. ${ }^{4}$ Prevalence of risk factors is also greater in low-socioeconomic status subjects in these countries. ${ }^{5}$

A major determinant of greater CVD mortality in lower socioeconomic status subjects is related to control of risk factors and quality of CVD-related preventive healthcare. ${ }^{5}{ }^{6}$ Low-socioeconomic status subjects have less access to CVD care and treatment, especially to good-quality primary care, which can reduce CVD risk factors by early detection and treatment. ${ }^{5}$ Analysis of nationally representative health examination surveys shows that two-thirds of people with diabetes and hypertension receive treatment in high-income countries such as the USA, while the coverage is $<50 \%$ in low-income and middle-income countries and the lowest rates are in rural regions in Sub-Saharan Africa ${ }^{1}$ and India. ${ }^{8}$ Studies in some low-income and middle-income countries have reported lower awareness, treatment and control of various CVD risk factors among low-socioeconomic status subjects compared with the middle and high. ${ }^{1}$

CVD are epidemic in India with high mortality rates. ${ }^{9}$ All the major CVD risk factors are also widely prevalent. ${ }^{9}$ Previous studies have reported that there are inequities in CVD risk factor prevalence related to socioeconomic status, especially educational status (ES). ${ }^{10}$ Subjects with lower educational (socioeconomic) status have higher smoking and tobacco use and consume an unhealthier diet. ${ }^{1-13}$ Prevalence of metabolic risk factors (obesity, diabetes and metabolic syndrome) is significantly greater in highsocioeconomic subjects, although hypertension prevalence is similar across the various socioeconomic groups. ${ }^{10}$ Studies have also reported lower awareness, treatment and control of hypertension among rural subjects compared with urban subjects. ${ }^{14}$ However, there is no study from India and other low-income or lower-middle-income countries that evaluated the association of treatment and control of multiple CVD risk factors such as hypertension, high cholesterol, diabetes and smoking with socioeconomic status. Therefore, to determine the prevalence of education status-related disparities in awareness, treatment and control of cardiometabolic risk factors (hypertension, diabetes, hypercholesterolaemia) and smoking/tobacco quit rates, we performed an epidemiological study among urban subjects in India. 


\section{METHODS}

A multisite study to identify prevalence of cardiovascular risk factors and their sociodemographic determinants was performed among urban subjects in India. Rationale for the study has been reported. ${ }^{9}$ The study case report form was developed according to recommendations of the WHO. ${ }^{15}$

The study data were collected in the years 2006-2010 at 11 cities in different geographic regions of the country as reported earlier. ${ }^{16}$ Simple cluster sampling was performed at each site. A middle-class location was identified at each city. This was based on municipal classification and derived from cost of land, type of housing, public facilities (roads, sanitation, water supply, electricity, gas supply), and educational and medical facilities. This intracity municipal classification is used to calculate the District Level Committee rates, which is a numerical value and can be used to classify locations into slums, low class, low-middle class, middle class, upper-middle class and upper class (see online supplementary table S1). More details are available at respective government websites, for example, for Jaipur at http://igrs. rajasthan.gov.in/images/pdf/SR1-Jaipur.pdf. ${ }^{17}$ Similar rates are available for almost all municipalities in cities that have been included (Madurai, Nagpur, Ahmedabad, Jaipur, Chandigarh, Lucknow, Patna). ${ }^{18}$ Local rates were available with the investigators at other cities (Bikaner, Jammu, Belguam, Dibrugarh). We invited 800-1000 subjects in each location to ensure participation of at least 500 subjects at each site according to WHO. ${ }^{15}$ Accordingly, the required sample size for giving an $85 \%$ chance of recognising a specified difference in mean values between two populations, significant at 5\% level (two-tailed test) with SD of individual values of 5.0 and a difference in mean values of 1.0 , requires a sample size of $470 .{ }^{15}$ Similarly, to have an $85 \%$ chance of recognising a specified difference in rates $(1-\beta$ power) between two populations, significant at the 5\% level (two-tailed test) and the estimated true rates in first population of $10 \%$ and in the second population of $5 \%$, requires a sample size of $490 .{ }^{15}$ We estimated a response rate of $70 \%$ as reported in previous studies at similar locations to arrive at the targeted sample size at each location. ${ }^{19}$ At each site a uniform protocol of recruitment was followed. The surveys were preceded by meetings with community leaders to ensure good participation. Subjects were invited in fasting state to a community centre or medical centre within each locality either twice or thrice a week depending upon the investigator's schedule. We invited all men and women $\geq 20$ years of age for interview and examination living within the locality for last 1 year. Subjects who did not provide an informed consent, were ill with terminal diseases and unable to visit the community centre or $<20$ years were excluded as reported earlier. ${ }^{16}$

The study case report form was filled after details were inquired from the subject. Apart from demographic history, details of socioeconomic status based on ES and years of formal education, type of family, any major previous illnesses, history of known hypertension, diabetes, lipid abnormalities and CVD were inquired. Details of smoking or tobacco use, alcohol intake, dietary fat, fruits and vegetables and physical activity were obtained as previously reported. ${ }^{16}$ Height, weight, waist and hip circumference and blood pressure were measured using standard WHO guidelines. ${ }^{15}$ Fasting blood sample was obtained from all individuals after $8-10 \mathrm{~h}$ fast. Blood glucose was measured at the local biochemistry facility while blood for cholesterol, cholesterol lipoproteins and triglycerides estimation was transported under dry ice to the national referral laboratory (http://www.thyrocare.com) at Mumbai, India, where tests were performed using uniform methodology.

Diagnostic criteria: ES was used as a proxy for socioeconomic status. ${ }^{4}$ Studies from India have reported good correlation of ES with socioeconomic status. ${ }^{2021}$ ES subjects were grouped as low (illiterate and $<10$ years of formal education), medium (1014 years of education) and high ( $\geq 15$ years of education). Smokers included subjects who smoked cigarettes, bidis or other non-smoked forms of tobacco daily, ex-smokers were subjects who had smoked for at least 1 year and had stopped more than a year ago. Users of other forms of tobacco (oral, nasal) were classified as smokeless tobacco use. Hypertension was diagnosed when systolic blood pressure was $\geq 140 \mathrm{~mm} \mathrm{Hg}$ and/or diastolic $\geq 90 \mathrm{~mm} \mathrm{Hg}$ or a person was a known hypertensive. Hypercholesterolaemia was defined by the presence of high total cholesterol $(\geq 200 \mathrm{mg} / \mathrm{dL}$ ). Diabetes was diagnosed on the basis of either history of known diabetes on treatment or fasting glucose $\geq 126 \mathrm{mg} / \mathrm{dL}$ as reported earlier. ${ }^{16}$ Awareness of hypertension, diabetes or hypercholesterolaemia was defined when the subject was aware of the risk factor. Treatment includes pharmacological therapy and control levels were diagnosed according to standard guidelines (blood pressure systolic $<140$ and diastolic $<90 \mathrm{~mm} \mathrm{Hg}$, fasting glucose $<126 \mathrm{mg} / \mathrm{dL}$ and total cholesterol $<200 \mathrm{mg} / \mathrm{dL}$ ).

Statistical analyses: All the data were computerised and entered into an SPSS database (V.10.0, SPSS, Chicago). More than $90 \%$ data for various variables were available, and in about $85 \%$ subjects data for all the variables were available. Numerical
Figure 1 Prevalence of major cardiovascular risk factors in men and women in the study cohort (age adjusted). HDL, high-density lipoprotein.

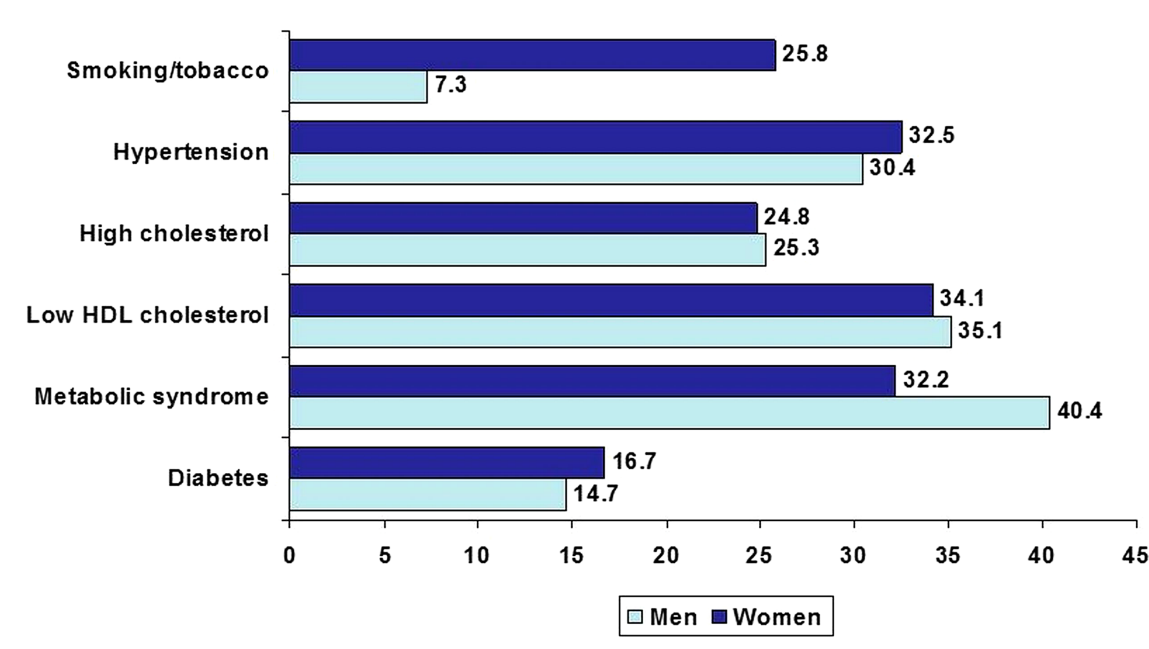

Gupta R, et al. Heart Asia 2015;7:1-6. doi:10.1136/heartasia-2014-010551 
variables are reported as means $\pm 1 \mathrm{SD}$ and categorical variables as per cent. Descriptive statistics are reported. Age adjustment was performed using direct method with 2001 Indian census population as reference or standard (India adult population in 2001 is provided in online supplementary table S1). Direct age adjustment is performed using the reference population categories as weights to form weighted averages for both populations using the formula: adjusted rate $=$ rate $\times \mathrm{N}$ in standard/total $\mathrm{N}$ in standard. ${ }^{22}$ To use this method of adjusted rates, the knowledge of specific rates for each category in the populations to be adjusted and the frequencies in the reference population for the factor being adjusted should be available. ${ }^{22}$ Prevalence of risk factors in the study population and in various groups has been reported as per cent and 95\% CIs. Awareness, treatment and control status of hypertension, hypercholesterolaemia and diabetes in various ES groups have been determined. Intergroup comparison was performed using $\chi^{2}$ test. Trends in prevalence, awareness, treatment and control of risk factors in various educational groups were determined using Mantel-Haenszel $\chi^{2}$ test for trend. $\mathrm{p}$ values of $<0.05$ have been considered significant.

\section{RESULTS}

The study was performed at 11 cities located in different geographic regions of India. ${ }^{16}$ In total, 6198 subjects (3426 men, 2772 women) of the targeted 9900 subjects were evaluated (response 62\%). Recruitment at individual sites and data for social and demographic characteristics in men and women have been reported. ${ }^{16}$ Men were slightly older than women, and there was no significant difference across various age groups. Low ES ( $<10$ years of formal education) was more among women $(47.6 \%)$ compared with men $(22.3 \%)$, and the majority of subjects belonged to middle ES. More than half of all men and women lived in joint families, and $85.6 \%$ were married.

Prevalence of major cardiovascular risk factors in men and women is shown in figure 1 . There is a moderately high prevalence of hypertension, diabetes, hypercholesterolaemia (total cholesterol $\geq 200 \mathrm{mg} / \mathrm{dL}$ ), low high-density lipoprotein cholesterol and metabolic syndrome. Prevalence of smoking/tobacco use is low, especially in women. In low-ES, medium-ES and high-ES groups, respectively, age-adjusted prevalence of hypertension was in $31.8 \%, 29.5 \%$ and $34.1 \%$, diabetes in $14.5 \%$, $15.3 \%$ and $14.3 \%$, hypercholesterolaemia in $24.0 \%, 23.9 \%$ and $27.3 \%$, and smoking/tobacco use in $24.3 \%, 14.4 \%$ and $19.0 \%$.

Awareness, treatment and control rates (per cent, 95\% CI), respectively, in subjects with hypertension were 55.3 (53.1 to 57.5), 36.5 (34.4 to 38.6) and 28.2 (26.2 to 30.2); diabetes 65.5 (62.5 to 68.5), 51.3 (48.1 to 54.4) and 29.6 (26.7 to 32.5); hypercholesterolaemia 19.3 (17.3 to 21.2), 9.3 (7.8 to $10.7)$ and 4.9 (3.8 to 6.0) and smoking quit rates were $3.3(2.3$ to 4.3). The awareness, treatment and control rates (\%) of hypertension, diabetes and hypercholesterolaemia in men and women are shown in table 1 . Awareness of hypertension is similar in men and women while diabetes and hypercholesterolaemia awareness, treatment and control are lower in women (figure 2).

In low-educational, medium-educational and high-educational groups, respectively, increasing trends with ES are observed for awareness of hypertension $(30.7,37.8,47.0)(p=0.047)$, diabetes $(47.2,51.5,56.4)(\mathrm{p}=0.024)$ and hypercholesterolaemia $(8.9,22.4,18.4)(\mathrm{p}=0.520)$. Increasing trends are also observed for hypertension treatment $(24.3,29.2,35.5, \mathrm{p}=0.046)$ and control $(7.8,11.6,15.5, \mathrm{p}=0.005)$; diabetes treatment (38.3, 41.3, 46.0, $\mathrm{p}=0.051)$ and control $(18.3,15.3,22.8, \mathrm{p}=0.593)$; and hypercholesterolaemia treatment $(4.1,6.2,7.9, \mathrm{p}=0.039)$

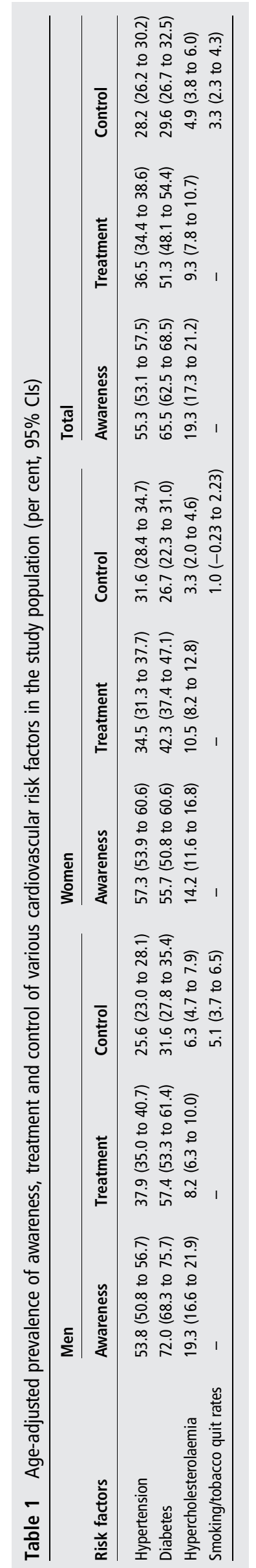


Figure 2 Age-adjusted awareness, treatment and control rates (\%) of hypertension, diabetes and hypercholesterolaemia in men and women. $\square$ Awareness $\square$ Treatment $\square$ Control



and control $(2.8,3.2,6.9, \mathrm{p}=0.297)$ as well as for smoking quit rates $(1.6,2.8,5.5, \mathrm{p}=0.139)$ (figure 3$)$. Trends for awareness, treatment and control of various risk factors were similar in men and women (table 2).

\section{DISCUSSION}

Lower socioeconomic status is associated with greater all-cause and cardiovascular mortality in India. ${ }^{10} 11$ Our study shows that there are significant ES-related disparities in awareness, treatment and control of major cardiovascular risk factors among middle-class urban subjects in India. Within the urban middle class, better educated men and women with hypertension, diabetes or hypercholesterolaemia are more aware of these risk factors and have better status of treatment and control compared with lower ES subjects.

Control of major cardiovascular risk factors is important for primary prevention of CVD. Extensive research has demonstrated that control of high blood pressure, high cholesterol levels and diabetes and smoking cessation leads to lower incidence of coronary heart disease and strokes. ${ }^{23}$ Better awareness of the disease and adequate treatment is important for control to targets. There is lower awareness, treatment and control of hypertension, diabetes and hypercholesterolaemia in our study subjects compared with studies from the USA and Europe. The NHANES studies have reported a secular increase in awareness, treatment and control of hypertension over the last 20 years, and the current rates in the USA are $>80 \% .^{24}$ Diabetes


Figure 3 Awareness, treatment and control of various risk factors (hypertension, diabetes, hypercholesterolaemia) and smoking quit rates in low-ES, medium-ES and high-ES groups (\%). Significantly increasing trends with ES are observed for awareness, treatment and control rates of all the risk factors (Mantel-Haenszel $\chi^{2}$ for trend, $p<0.05$ ). 
Table 2 Age-adjusted awareness, treatment and control status of various cardiovascular risk factors according to ES in men and women (per cent, $95 \% \mathrm{Cls}$ )

\begin{tabular}{|c|c|c|c|c|c|c|c|}
\hline \multirow[b]{2}{*}{ Risk factors } & \multirow{2}{*}{$\begin{array}{l}\text { Educational } \\
\text { status (years) }\end{array}$} & \multicolumn{3}{|l|}{ Men } & \multicolumn{3}{|l|}{ Women } \\
\hline & & Awareness* & Treatment* & Control* & Awareness* & Treatment* & Control* \\
\hline Hypertension & $\begin{array}{l}\leq 10 \\
11-15 \\
>15\end{array}$ & $\begin{array}{l}26.6 \text { (20.3 to } 32.9) \\
34.2 \text { (30.8 to } 37.6) \\
45.5 \text { (40.8 to } 15.2)\end{array}$ & $\begin{array}{l}25.0 \text { (18.8 to } 31.2) \\
26.3 \text { (23.1 to } 29.5) \\
33.5(29.0 \text { to } 37.9)\end{array}$ & $\begin{array}{c}9.5(5.3 \text { to } 13.7) \\
10(7.8 \text { to } 12.1) \\
15.4(12.0 \text { to } 18.8)\end{array}$ & $\begin{array}{l}32.8 \text { (28.1 to } 37.5) \\
43.3(38.8 \text { to } 47.8) \\
51.7 \text { (43.6 to } 59.8)\end{array}$ & $\begin{array}{l}24.0 \text { (19.7 to } 28.2 \\
33.8(29.5 \text { to } 38.1) \\
41.4(33.4 \text { to } 49.4)\end{array}$ & $\begin{array}{l}9.5(6.6 \text { to } 12.4) \\
14.4(11.2 \text { to } 17.5) \\
15.8(9.8 \text { to } 21.7)\end{array}$ \\
\hline Diabetes & $\begin{array}{l}\leq 10 \\
11-15 \\
>15\end{array}$ & $\begin{array}{l}49.5 \text { (39.8 to } 59.1) \\
51.5 \text { (46.8 to } 56.2) \\
55.9(49.3 \text { to } 62.0)\end{array}$ & $\begin{array}{l}39.0(29.6 \text { to } 48.4) \\
41.4(36.8 \text { to } 46.0) \\
45.9(39.3 \text { to } 52.5)\end{array}$ & $\begin{array}{r}18.4(10.9 \text { to } 25.9) \\
15(11.6 \text { to } 18.3) \\
21.8(16.3 \text { to } 27.3)\end{array}$ & $\begin{array}{l}45.8(38.3 \text { to } 53.3) \\
50.4(43.8 \text { to } 56.9) \\
57.9(46.2 \text { to } 69.5)\end{array}$ & $\begin{array}{l}37.6(30.3 \text { to } 44.9) \\
41.1(34.7 \text { to } 47.5) \\
46.3(34.5 \text { to } 58.1)\end{array}$ & $\begin{array}{l}18.2(12.4 \text { to } 24.0) \\
15.6(10.8 \text { to } 20.3) \\
26.0(15.6 \text { to } 36.3)\end{array}$ \\
\hline \multirow[t]{2}{*}{ Hypercholesterolaemia } & $\leq 10$ & 12.3 (6.4 to 18.1 ) & $3.3(0.12$ to 6.48$)$ & 7.4 (2.7 to 12.1$)$ & 7.3 (4.2 to 10.4 ) & 4.4 (1.9 to 6.8$)$ & 1.4 (0.0 to 2.8 ) \\
\hline & $>15$ & 19.6 (15.8 to 23.8$)$ & 7.6 (4.8 to 10.4 ) & 7.6 (4.8 to 10.4 ) & 15.6 (9.5 to 21.7$)$ & 8.8 (4.0 to 13.5$)$ & $4.4(0.95$ to 7.85$)$ \\
\hline \multirow[t]{3}{*}{ Smoking quit rates } & $\leq 10$ & - & - & 3.7 (1.9 to 5.5$)$ & - & - & $0.5(0.02$ to 0.98$)$ \\
\hline & $11-15$ & - & - & 4.2 (3.3 to 5.1 ) & - & - & $0.9(0.37$ to 1.43$)$ \\
\hline & $>15$ & - & - & $7.4(5.7$ to 9.0$)$ & - & - & $1.0(0.02$ to 1.98$)$ \\
\hline
\end{tabular}

awareness is $>90 \%$ in most high-income countries and hypercholesterolaemia awareness $>50 \% .^{25}$ Decline in smoking and alcohol intake as well as control of major cardiovascular risk factors related to improved ES account for the significant decline in cardiovascular mortality in Europe and North America in the last 50 years. ${ }^{5}$

Although in our population of middle-class urban subjects there is better awareness of hypertension and diabetes (figure 2) compared with other studies from India, ${ }^{14}$ the hypercholesterolaemia awareness is extremely low. This may be related to low prevalence of this abnormality as well as lack of public education programme focused on cardiovascular risk factors. Education-related disparities are, however, observed in awareness, treatment and control among all these groups (figure 3) with better educated subjects showing greater awareness, treatment and control of all the studied risk factors. Socioeconomic disparities are well known in chronic disease awareness and management. ${ }^{1}$ The PURE study has reported that among patients with CVD in South Asia there is lower use of all the four evidence-based medicines (antiplatelets, $\beta$-blockers, angiotensin-converting enzyme inhibitors/angiotensin receptor blockers and statins). ${ }^{26}{ }^{27}$ Subjects with lower ES and low wealth index had significantly lower use of these drugs. ${ }^{27}$ Adoption of healthy lifestyles in patients with known cardiovascular disease was also lower in low-income countries in the PURE study compared with high-income and middle-income countries. ${ }^{28}$ Improving trends in uptake of secondary prevention therapies and healthier lifestyles are reported from upperincome and middle-income countries. ${ }^{15}$ In the present study, smoking quit rates are low despite universal awareness. Smoking quit rates approach $40 \%$ in many high-income countries. ${ }^{6}$ Better quit rates among more educated subjects indicate importance of general literacy in smoking cessation programmes. Similar observations have been reported from developed countries as well as from serial National Family Health Surveys in India. ${ }^{12}$

Limitations of the study are biases introduced because of sampling, non-representation of the Indian population, inclusion of only urban subjects, low response rates, measurement techniques and failure to correct for regression-dilution. However, many of the limitations are inherent in a cross-sectional epidemiological study ${ }^{15}$ and the data are therefore subject to similar biases. Urban locations are hotbeds of CVD epidemic in
India, ${ }^{29}$ and the present study is, therefore, important. Moreover, similar methodology is used in the previous Indian studies and the present data are similarly representative. ${ }^{29}$ Second, multiple parameters could be used to assess socioeconomic status. Education is a summary measure of early-life experiences, childhood literacy (surrogate for health literacy), adult occupation and incomes and also provides coping abilities. ${ }^{4}$ This variable has been used the most in cardiovascular epidemiological studies. ${ }^{4} 5$ We, and others, have previously reported that in India there is a significant correlation of educational level with occupation, housing, neighbourhood measures and social status. ${ }^{10} 2021$ Third, low response rate in the study $(62 \%)$ is also a concern and it is possible that those excluded were more or less healthy compared with the study subjects; however, these response rates are similar to other populationbased studies in India and elsewhere and are within acceptable limits. ${ }^{30}$ Finally, there are multiple determinants of the inverse social gradient for cardiovascular risk factor awareness, treatment and control. We have not analysed the 'causes of the causes' or the societal factors that lead to greater cardiovascular risk in lower ES subjects. The societal causes include social organisation, social support, cohesion, social exclusion, lifecourse social gradient, unemployment, environment at work, transport, and so on, and cannot comment on these. ${ }^{31}$ On the other hand, strengths of the study include nationwide scope of the study, adequate representation of men and women of various ES groups and study of multiple risk factors.

In conclusion, this study shows that lower ES subjects in India have lower awareness, treatment and control of major cardiovascular risk factors. Whether these disparities lead to greater adverse outcomes in this group of subjects is a matter of further study. Meanwhile, it is important to focus on improving status of cardiovascular risk factor control through promoting awareness among the general population and to enhance treatment status by proper physician education.

Acknowledgements This study was funded by South Asian Society of Atherosclerosis and Thrombosis, Bangalore, India, and Minneapolis, USA. The funders had no role in study design, data collection and analysis, decision to publish or preparation of the manuscript.

Contributors $R G, A G$ and $P C D$ designed and supervised the study. $R G, A G$ and BKG were also involved in data collection and supervision of the study. KKS and RRG were involved in data management and analyses. All authors have significantly contributed to drafting of the article. 
Competing interests None.

Patient consent Obtained.

Ethics approval Institutional Ethics Committee, Fortis Escorts Hospital, Jaipur, India.

Provenance and peer review Not commissioned; externally peer reviewed.

\section{REFERENCES}

1 DiCesare $\mathrm{M}$, Khang $\mathrm{Y}-\mathrm{H}$, Asaria $\mathrm{P}$, et al. on behalf of the Lancet NCD Action Group. Inequities in non-communicable diseases and effective responses. Lancet 2013;381:585-97.

2 Lozano R, Naghavi M, Foreman K, et al. Global and regional mortality from 235 causes of death for 20 age-groups in 1990 and 2010: a systematic analysis for the Global Burden of Disease Study 2010. Lancet 2012;380:2095-128.

3 Yusuf S, Rangarajan S, Teo K, et al. on behalf of PURE Investigators. Cardiovascular risk and events in 17 low-, middle- and high-income countries. $N$ Engl J Med 2014;371:818-27.

4 Kaplan GA, Keil JE. Socioeconomic factors and cardiovascular disease: a review of the literature. Circulation 1993:88:1973-98.

5 Harper S, Lynch J, Davey Smith G. Social determinants and the decline of cardiovascular diseases: understanding the links. Annu Rev Public Health 2011;32:39-69.

6 Atun R, Jaffar $S$, Nishtar $S$, et al. Improving responsiveness of health systems to non-communicable diseases. Lancet 2013;381:690-7.

7 Hogerzeil HV, Liberman J, Wirtz VJ, et al. on behalf of the Lancet NCD Action Group. Promotion of access to essential medicines for non-communicable diseases: practical implications of the UN political declaration. Lancet 2013;381:680-9.

8 World Health Organization. Global status report on non communicable diseases 2010. Geneva: World Health Organization, 2011.

9 Gupta R, Guptha S, Sharma KK, et al. Regional variations in cardiovascular risk factors in India: India Heart Watch. World J Cardiol 2012;4:112-20.

10 Prabhakaran D, Jeemon P, Reddy KS. Poverty and cardiovascular disease in India: do we need more evidence for action? Int J Epidemiol 2013;42:1431-5.

11 Gupta R, Gupta KD. Coronary heart disease in low socioeconomic status subjects in India: an evolving epidemic. Indian Heart J 2009;61:358-67.

12 Bhan N, Srivastava S, Agrawal S, et al. Are socioeconomic disparities in tobacco consumption increasing in India? A repeated cross-sectional multilevel analysis. BMJ Open 2012;2:e001348.

13 Vellakkal S, Subramanian SV, Millet C, et al. Socioeconomic inequities in non-communicable diseases prevalence in India: disparities between self-reported diagnosis and standardized measures. PLOS ONE 2013;8:e68219.

14 Gupta R, Gupta N. Hypertension epidemiology in the 21st century India. J Prev Cardiol 2013;2:350-5.
15 Luepkar RV, Evans A, McKeigue P, et al. Cardiovascular survey methods. 3rd edn. Geneva: World Health Organization, 2002.

16 Gupta R, Deedwania PC, Sharma KK, et al. Association of education, occupation and socioeconomic status with cardiovascular risk factors in Asian Indians: a cross-sectional study. PLOS ONE 2012;7:e44098.

17 Office of Sub-Registrar-1 Jaipur. Current report of DLC rate (residential/commercial). http://igrs.rajasthan.gov.in/images/pdf/SR1-Jaipur.pdf (accessed 7 September 2014).

18 Mathur OP, Thakur D, Rajadhyaksha N, et al. Urban property tax potential in India. New Delhi: National Institute of Public Finance and Policy, 2009.

19 Gupta R, Gupta VP, Sarna M, et al. Prevalence of coronary heart disease and risk factors in an urban Indian population: Jaipur Heart Watch-2. Indian Heart $J$ 2002;54:59-66.

20 Gupta R, Gupta VP, Ahluwalia NS. Educational status, coronary heart disease and coronary risk factor prevalence in a rural population of India. BMJ 1994;309:1332-6.

21 Agrawal S, Karan A, Selvaraj $S$, et al. Socioeconomic patterning of tobacco use in Indian states. Int J Tuberc Lung Dis 2013;17:1110-17.

22 Dawson B, Trapp RG. Summarizing data and presenting data in tables and graphs. In: Basic and clinical biostatistics. 4th edn. Boston: McGraw Hill International Edition, 2004:23-60.

23 Gaziano JM, Ridker PM, Libby P. Primary and secondary prevention of coronary heart disease. In: Bonow RO, Mann DL, Zipes DP, Libby P, eds. Braunwald's heart disease. 9th edn. Philadelphia: Saunders, 2012:1010-35.

24 Guo F, He D, Zhan W, et al. Trends in prevalence, awareness, management and control of hypertension among United States adults, 1999 to 2010. J Am Coll Cardiol 2012;60:599-606.

25 Mackenbach JP, Stribu I, Roskam AJ, et al. for the European Union Working Group on Socioeconomic Inequalities in Health. Socioeconomic inequalities in health in 22 European countries. N Engl J Med 2008:358:2468-81.

26 Yusuf S, Islam S, Chow CK, et al. Prospective Urban Rural Epidemiology (PURE) Study Investigators. Low use of secondary prevention medications for cardiovascular disease in the community in 17 high, middle and low income countries (The PURE Study). Lancet 2011;378:1231-43.

27 Gupta R, Mony P, Shankar K, et al. Socioeconomic status and cardiovascular secondary prevention therapies in South Asia: the PURE Study. Eur J Prev Cardiol 2014. pii:2047487314540386.

28 Teo KK, Lear S, Islam S, et al. PURE Investigators. Prevalence of a healthy lifestyle among individuals with cardiovascular disease in high-, middle-, and low income countries: the Prospective Urban Rural Epidemiology (PURE) study. JAMA 2013;309:1613-21.

29 Gupta R, Gupta VP. Meta-analysis of coronary heart disease prevalence in India. Indian Heart J 1996:48:241-5.

30 Johnson TP, Wislar JS. Response rates and non-response errors in surveys. JAMA 2012;307:1805-6.

31 Marmot M, Friel S, Bell R, et al. Commission on Social Determinants of Health. Closing the gap in a generation: health equity through action on the social determinants of health. Lancet 2008;372:1661-9. 